\title{
Efficient Eigenspace-Based Array Signal Processing Using Multiple Shift-Invariant Subarrays
}

\author{
Shiann-Jeng Yu and Ju-Hong Lee
}

\begin{abstract}
This paper deals with the construction of eigensubspaces for adaptive array signal processing. An efficient technique for extracting the eigensubspaces spanned by the data vector received by an $N$-element adaptive array is presented. We first decompose the original array into several subarrays with multiple shift invariances and find the eigensubspaces corresponding to each of the subarrays. By solving a least-squares (LS) or total least-squares (TLS) problem, the signal and noise subspaces corresponding to the original array can be found from the eigensubspaces spanned by the subarray data vectors. Hence, there is no need to perform the eigenvalue decomposition of the $N$ $\times \mathbf{N}$ correlation matrix of the received data vector. The proposed technique significantly reduces the required computational complexity as compared to the conventional eigenspace-based (ESB) methods. In conjunction with the spatial smoothing scheme or a proposed cross-correlation method, this technique can also deal with the case of coherent signals. The effectiveness of the proposed technique is demonstrated by several computer simulations.
\end{abstract}

Index Terms-Adaptive arrays, array signal processing.

\section{INTRODUCTION}

$\mathbf{E}$ IGENSPACE-BASED (ESB) methods have been widely used for processing the data vector received by an $N$ element adaptive array. The well-known MUSIC algorithm [1] utilizes the property that noise subspace is orthogonal to signal direction vectors to estimate the signal bearings. The ESPRIT algorithm [2] exploits the shift-invariant property between two identical subarrays and employs the signal subspaces obtained from the subarrays to estimate the desired signal parameters. Later, Swindlehurst et al. [3] presented a multiple invariance ESPRIT (MI ESPRIT) to enhance the performance of the ESPRIT algorithm by employing the property of multiple shiftinvariant subarrays. The design of adaptive array beamformers based on the use of the signal subspace was presented by Chang and Yeh [4]. It has been shown that the ESB adaptive beamforming possesses the advantage of faster convergence speed over conventional adaptive beamforming. In the area of partially adaptive array beamforming, Van Been [5] has shown that an ESB partially adaptive beamformer can provide almost the same performance as its counterpart with full adaptivity. Moreover, Haimovich and Bar-Ness [6] considered

Manuscript received March 21, 1995; revised July 29, 1998. This work was supported by the National Science Council under Grant NSC85-2213-E002008 .

The authors are with the Department of Electrical Engineering, National Taiwan University, Taipei, 106 Taiwan.

Publisher Item Identifier S 0018-926X(99)02474-6. the interference cancellers for receiving the desired signal and found that an interference canceller designed based on the use of eigenspace property provides better array performance and faster convergence speed. In the implementation of the above ESB methods, the computational complexity is $O\left(N^{3}\right)$ since an $N \times N$ eigenvalue decomposition (EVD) of the correlation matrix of the received data vector is generally required.

In the case of coherent signals, it has been shown [7] that the above methods cannot successfully process array signals for bearing estimation and adaptive beamforming. To tackle the coherent problem, Shan and Kailath [8] presented a spatial smoothing scheme to reduce the coherence between the signals for adaptive beamforming. This technique was later applied to bearing estimation [9], [10]. Recently, it was further extended to deal with the problem of estimating two-dimensional (2-D) angles of arrival in the presence of coherent signals [11]. Although the above techniques can alleviate the effect of signal coherence, they degrade the array performance since the degrees of freedom associated with the original array are reduced. Recently, a method was presented to solve the problem of the reduction in degrees of freedom [12], however, it requires an iterative nonlinear optimization procedure to estimate the noise subspace required for adaptive beamforming and is only suitable when the array is linearly periodic.

In this paper, we present an efficient technique for extracting the eigensubspaces of a correlation matrix of a data vector received by an $N$-element adaptive array with arbitrary geometry. The original adaptive array is partitioned into several subarrays with multiple shift-invariant property. Utilizing the multiple shift invariances, we show that the problem of finding the eigensubspaces corresponding to the original array can be formulated as a least-squares (LS) or total least-squares (TLS) problem. As a result, the signal and noise subspaces corresponding to the original array can be found from the eigensubspaces spanned by the subarray data vectors by solving the resulting LS or TLS problem. Therefore, the $N \times N$ EVD of the correlation matrix, which is generally required by conventional ESB methods, can be avoided. In conjunction with the spatial smoothing scheme for bearing estimation or a proposed cross-correlation method for interference cancellation, the proposed technique can also deal with the case of coherent signals without sacrificing the available degrees of freedom and requiring any nonlinear optimization process. Computer simulations show that the proposed technique is effective whether the original array 
is one-dimensional (1-D) or 2-D. Moreover, it demonstrates better performance than the existing methods.

\section{ARray Signal Processing Based on Eigenstructure}

1) The Eigenstructure of A. Data Correlation Matrix: Consider an $N$-element array with arbitrary geometry. Assume that $J$ far-field narrow-band signal sources are impinging on the array from different directions. Then the received data of the $i$ th array element is given by

$$
x_{i}(t)=\sum_{j=1}^{J} a_{i}\left(\theta_{j}\right) s_{j}(t)+n_{i}(t)
$$

where $a_{i}\left(\theta_{j}\right)$ is a complex scaler representing the gain and phase shift of the $j$ th signal $s_{j}(t)$ received by the $i$ th array element. $n_{i}(t)$ represents the received additive white Gaussian noise with mean zero and variance $\sigma_{n}^{2}$. The corresponding data vector received by the $N$-element array can be written as

$$
\begin{aligned}
X(t) & =\left[\begin{array}{ll}
a\left(\theta_{1}\right) & a\left(\theta_{2}\right) \cdots a\left(\theta_{J}\right)
\end{array}\right] S(t)+N(t) \\
& =\mathbf{A}(\theta) S(t)+N(t)
\end{aligned}
$$

where the direction vector of the $j$ th source is $a\left(\theta_{j}\right)=$ $\left[a_{1}\left(\theta_{j}\right) \quad a_{2}\left(\theta_{j}\right) \cdots a_{N}\left(\theta_{j}\right)\right]^{T}$, the signal source vector is $S(t)=\left[\begin{array}{ll}s_{1}(t) & s_{2}(t) \cdots s_{J}(t)\end{array}\right]^{T}$, and the noise vector is $N(t)=\left[\begin{array}{lll}n_{1}(t) & n_{2}(t) \cdots n_{N}(t)\end{array}\right]^{T} . S(t)$ and $N(t)$ are uncorrelated. The superscript $T$ denotes transpose operation. Then the ensemble correlation matrix of $X(t)$ is given by

$$
\mathbf{R}_{x}=E\left\{X(t) X^{H}(t)\right\}=\mathbf{A}(\theta) \mathbf{S A}^{H}(\theta)+\sigma_{n}^{2} \mathbf{I}
$$

where the superscript $H$ denotes the complex conjugate transpose. $\boldsymbol{S}=E\left\{S(t) S^{H}(t)\right\}$ has rank $K \leq J$.

In the incoherent signal environment, $K=J$ and the EVD of (3) can be expressed as

$$
\mathbf{R}_{x}=\sum_{i=1}^{N} \lambda_{i} e_{i} e_{i}^{H}=\mathbf{E}_{s} \Lambda_{s} \mathbf{E}_{s}^{H}+\mathbf{E}_{n} \Lambda_{n} \mathbf{E}_{n}^{H}
$$

where $\lambda_{1}>\lambda_{2}>\cdots>\lambda_{K}>\lambda_{K+1}=\cdots=\lambda_{N}=\sigma_{n}^{2}$ and $e_{i}$ is the eigenvector associated with $\lambda_{i}$. The basis matrix $\mathbf{E}_{s}=\left[\begin{array}{llll}e_{1} & e_{2} & \cdots & e_{K}\end{array}\right]$ spans the signal subspace, while the basis matrix $\mathbf{E}_{n}=\left[\begin{array}{llll}e_{K+1} & e_{K+2} & \cdots & e_{N}\end{array}\right]$ spans the noise subspace, which is orthogonal to the signal subspace. It has been shown in [1] that $\mathbf{E}_{s}$ and $\mathbf{A}(\theta)$ span the same signal subspace. On the other hand, $K<J$ in the coherent signal environment. For this case, the rank of $\mathbf{E}_{s}$ is less than $J$. Hence, $\mathbf{E}_{s}$ does not have enough degrees of freedom to span the same signal subspace as that spanned by $\mathbf{A}(\theta)$.

To tackle the coherent problem, a spatial smoothing technique is presented in [8] at the price of reducing the effective size of the original array. By partitioning the array into several overlapping subarrays with the same size greater than $J$, we obtain a smoothed correlation matrix which is the average of the data correlation matrices of the subarrays. It has been shown in [8]-[11] that the smoothed correlation matrix possesses exactly the same form as the data correlation matrix for an incoherent case. Therefore, using the smoothed correlation matrix to replace $\mathbf{R}_{x}$ can destroy the coherence between the signal sources and work successfully regardless of the coherent signals.

\section{B. Principle of Eigenspace-Based Array-Signal Processing}

1) Eigenspace-Based Interference Cancellation: Consider the case of incoherent signals. For convenience, let $s_{J}(t)$ represent the desired signal with direction vector $S_{d}=a\left(\theta_{J}\right)$ and the other $J-1$ signals be interferers. Without loss of generality, we assume that the desired signal is impinging on the array from the broadside, i.e., $S_{d}=\left[\begin{array}{llll}1 & 1 & \cdots & 1\end{array}\right]^{T}$. An interference canceller adjusts the weights such that the array has zero gain in the interference directions, while maintains a unit gain in the direction of the desired signal. The optimal weight vector of an interference canceller is obtained by solving the following minimization problem

$$
\begin{aligned}
& \text { Minimize } W^{H} \mathbf{R}_{x} W \\
& \text { Subject to } S_{d}^{H} W=1 \text { and } \mathbf{E}_{I}^{H} W=0
\end{aligned}
$$

where $\mathbf{E}_{I}=\left[\begin{array}{ll}a\left(\theta_{1}\right) & a\left(\theta_{2}\right) \cdots a\left(\theta_{J-1}\right)\end{array}\right]$ denotes the matrix containing the $J-1$ interference direction vectors. The optimal solution of (5) is given by [6]

$$
W_{o}=\frac{\overline{\mathbf{E}}_{I} \overline{\mathbf{E}}_{I}^{H} S_{d}}{S_{d}^{H} \overline{\mathbf{E}}_{I} \overline{\mathbf{E}}_{I}^{H} S_{d}},
$$

where the matrix $\overline{\mathbf{E}}_{I}$ has rank $=N-J+1$ and is given by

$$
\overline{\mathbf{E}}_{I}=\mathbf{I}-\mathbf{E}_{I}\left(\mathbf{E}_{I}^{H} \mathbf{E}_{I}\right)^{-1} \mathbf{E}_{I}^{H} .
$$

To obtain $\mathbf{E}_{I}$, it has been proposed in [6] and [12] to use a signal blocking matrix to remove the desired signal from the received data vector and then perform EVD on the correlation matrix of the signal-blocked data vector. Consider an $N \times(N-1)$ signal blocking matrix $\mathbf{B}$ given by [6]

$$
\mathbf{B}^{H}=\left[\begin{array}{rrrrrr}
1 & -1 & 0 & \cdots & 0 & 0 \\
0 & 1 & -1 & \cdots & 0 & 0 \\
\vdots & \vdots & \vdots & \vdots & \vdots & \vdots \\
0 & 0 & 0 & \cdots & 1 & -1
\end{array}\right] .
$$

Then the data vector $Y(t)=\mathbf{B}^{H} X(t)$ at the output of $\mathbf{B}$ does not contain the desired signal. The interference subspace or the noise subspace spanned by $Y(t)$ is obtained by performing the EVD on the correlation matrix of $Y(t)$.

2) Bearing Estimation: Consider the MUSIC algorithm for estimating signal bearings. It has been shown in [1] that $\mathbf{E}_{s}$ and $\mathbf{A}(\theta)$ span the same signal subspace. Each of the signal direction vectors is orthogonal to the noise subspace spanned by $\mathbf{E}_{n}$. Therefore, the MUSIC algorithm estimates the signal bearings by searching the peaks of the following spatial spectrum:

$$
E(\theta)=\frac{1}{S^{H}(\theta) \mathbf{E}_{n} \mathbf{E}_{n}^{H} S(\theta)}
$$

where $S(\theta)=\left[\begin{array}{ll}s_{1}(\theta) & s_{2}(\theta) \cdots s_{N}(\theta)\end{array}\right]^{T}$ with $s_{i}(\theta)=$ $\exp \left(j \kappa_{c} d_{i} \sin (\theta)\right)$ denoting the search vector function, $\kappa_{c}$ is the wavenumber of the signals, and $d_{i}$ is the position of the $i$ th sensor. 


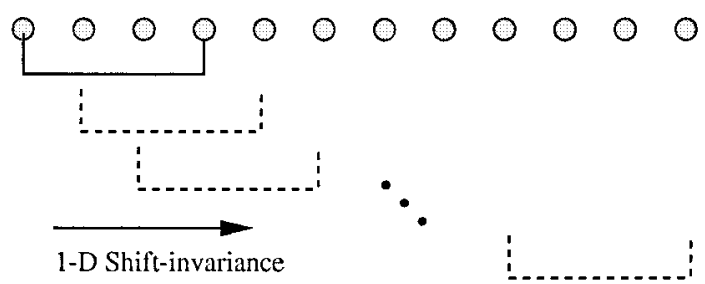

(a)

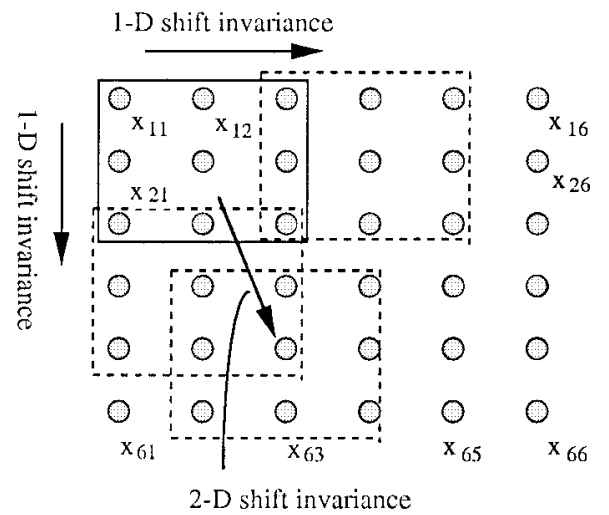

(b)

Fig. 1. Examples of arrays with multiple shift invariances. (a) The uniform linear array. (b) The uniform rectangular array.

\section{The Proposed Technique}

For an $N$-element adaptive array with arbitrary geometry, we partition it into several subarrays with multiple shift invariances. Fig. 1 plots the array scenarios considered. A uniform linear array (ULA) has 1-D shift invariance, while a uniform rectangular array (URA) has both 1-D and 2-D shift invariances. Let the $N$-element adaptive array be partitioned into $K$ nonoverlapped subarrays and $N_{k}>J$ be the number of array elements of the $k$ th subarray for $k=1,2, \cdots, K$.

First, consider the case of incoherent signals. Let $X_{k}(t)$ be the data vector received by the $k$ th subarray and its correlation matrix be denoted by $\mathbf{R}_{x k}=E\left\{X_{k}(t) \quad X_{k}^{H}(t)\right\}$. Performing the EVD on $\mathbf{R}_{x k}$, we obtain

$$
\mathbf{R}_{x k}=\mathbf{E}_{s k} \Lambda_{s k} \mathbf{E}_{s k}^{H}+\mathbf{E}_{n k} \Lambda_{n k} \mathbf{E}_{n k}^{H}
$$

where $\Lambda_{s k}=\operatorname{diag}\left\{\lambda_{k 11}, \lambda_{k 2}, \cdots, \lambda_{k J}\right\}$ contains the signal eigenvalues associated with the signal eigenvectors contained in $\mathbf{E}_{s k}=\left[e_{k 1}, e_{k 2}, \cdots, e_{k J}\right]$, while $\Lambda_{n k}=$ $\operatorname{diag}\left\{\lambda_{k(J+1)}, \lambda_{k(J+2)}, \cdots, \lambda_{k N_{k}}\right\}=\operatorname{diag}\left\{\sigma_{n}^{2}, \sigma_{n}^{2}, \cdots, \sigma_{n}^{2}\right\}$ contains the eigenvalues associated with the noise eigenvectors contained in $\mathbf{E}_{n k}=\left[e_{k(J+1)}, e_{k(J+2)}, \cdots, e_{k N_{k}}\right]$. Using $\mathbf{E}_{s k}$ and $\mathbf{E}_{n k}$, we construct the following two matrices:

$$
\mathbf{Q}_{1}=\left[\begin{array}{ccccc}
\mathbf{E}_{n 1} & \mathbf{O} & \cdots & \mathbf{O} & \mathbf{O} \\
\mathbf{O} & \mathbf{E}_{n 2} & \cdots & \mathbf{O} & \mathbf{O} \\
\mathbf{O} & \mathbf{O} & \cdots & \vdots & \vdots \\
\vdots & \vdots & \vdots & \mathbf{O} & \mathbf{O} \\
\mathbf{O} & \mathbf{O} & \cdots & \mathbf{E}_{n K-1} & \mathbf{O} \\
\mathbf{O} & \mathbf{O} & \cdots & \mathbf{O} & \mathbf{E}_{n K}
\end{array}\right]
$$

with size of $N \times(N-K J)$ and

$$
\mathbf{Q}_{2}=\left[\begin{array}{ccccc}
\mathbf{E}_{s 1} & \mathbf{O} & \cdots & \mathbf{O} & \mathbf{O} \\
\mathbf{O} & \mathbf{E}_{s 2} & \cdots & \mathbf{O} & \mathbf{O} \\
\mathbf{O} & \mathbf{O} & \cdots & \vdots & \vdots \\
\vdots & \vdots & \vdots & \mathbf{O} & \mathbf{O} \\
\mathbf{O} & \mathbf{O} & \cdots & \mathbf{E}_{s K-1} & \mathbf{O} \\
\mathbf{O} & \mathbf{O} & \cdots & \mathbf{O} & \mathbf{E}_{s K}
\end{array}\right]
$$

with size of $N \times K J$. It follows from (11) and (12) that $\mathbf{Q}_{1}^{H} \mathbf{Q}_{1}=\mathbf{I}, \mathbf{Q}_{2}^{H} \mathbf{Q}_{2}=\mathbf{I}$, and $\mathbf{Q}_{1}^{H} \mathbf{Q}_{2}=\mathbf{O}$, where $\mathbf{I}$ denotes the identity matrix and $\mathbf{O}$ the zero matrix. Furthermore, let $\mathbf{Q}=\left[\begin{array}{ll}\mathbf{Q}_{1} & \mathbf{Q}_{2}\end{array}\right]$, then it is easy to show that $\mathbf{Q Q}^{H}=\mathbf{Q}^{H} \mathbf{Q}=$ $\mathbf{I}$, i.e., $\mathbf{Q}$ is a unitary transformation matrix with rank $=N$.

From (11), we have the following theorem.

Theorem 1: Each column of $\mathbf{Q}_{1}$ represents an eigenvector of $\mathbf{R}_{x}$ corresponding to the eigenvalue $\sigma_{n}^{2}$. In other words, each column of $\mathbf{Q}_{1}$ is also an eigenvector of $\mathbf{R}_{x}$.

Proof: For any column of $\mathbf{Q}_{1}$, it can be expressed as

$$
v=\left[0 \cdots 0 e_{k j}^{T} 0 \cdots 0\right]^{T}
$$

for $k=1,2, \cdots, K$, and $j=J+1, \cdots, N_{k}$. Using (3), we have

$$
\mathbf{R}_{x} v=\left(\mathbf{A}(\theta) \mathbf{S} \mathbf{A}^{H}(\theta)+\sigma_{n}^{2} \mathbf{I}\right) v .
$$

Since $e_{k j}$ denotes a noise eigenvector of $\mathbf{R}_{x k}$, it is orthogonal to the source direction vectors viewed by the $k$ th subarray. Hence, we can find that

$$
\begin{aligned}
v_{H} \mathbf{A}(\theta) & =\left[\begin{array}{lll}
0 \cdots 0 e_{k j}^{H} 0 \cdots 0 & \cdots
\end{array}\left[\begin{array}{c}
\mathbf{A}_{1}(\theta) \\
\vdots \\
\mathbf{A}_{k}(\theta) \\
\vdots \\
\mathbf{A}_{N}(\theta)
\end{array}\right]\right. \\
& =e_{k j}^{H} \mathbf{A}_{k}(\theta)=0
\end{aligned}
$$

where $\mathbf{A}_{k}(\theta)$ denotes the $N_{k} \times J$ matrix containing the signal direction vectors viewed by the kth subarray. Substituting (15) into (14) yields

$$
\mathbf{R}_{x} v=\sigma_{n}^{2} v .
$$

Equation (16) reveals that $v$ is also a noise eigenvector of $\mathbf{R}_{x}$. This completes the proof.

Theorem 1 shows that $N-K J$ distinct noise eigenvectors of $\mathbf{R}_{x}$ can be obtained from $\mathbf{R}_{x k}$. However, there still remains $(K-1) J$ noise eigenvectors and $J$ signal eigenvectors of $\mathbf{R}_{x}$ required to be determined. Since $\mathbf{Q}=\left[\begin{array}{ll}\mathbf{Q}_{1} & \mathbf{Q}_{2}\end{array}\right]$ is a unitary transformation matrix with rank $=N$, we note that the remaining $(K-1) J$ noise eigenvectors and $J$ signal eigenvectors must lie in the column span of $\mathbf{Q}_{2}$. Therefore, there exists two coefficient matrices $\mathbf{G}$ and $\mathbf{H}$ with sizes equal to $(K J) \times J$ and $(K J) \times((K-1) J)$, respectively, such that

$$
\tilde{\mathbf{E}}_{s}=\mathbf{Q}_{2} \mathbf{G}
$$

and

$$
\tilde{\mathbf{E}}_{n}=\left[\begin{array}{ll}
\mathbf{Q}_{1} & \mathbf{Q}_{2} \mathbf{H}
\end{array}\right]
$$


span the same signal and noise subspaces as those spanned by $\mathbf{E}_{s}$ and $\mathbf{E}_{n}$, respectively.

To compute these two coefficient matrices $\mathbf{G}$ and $\mathbf{H}$, we present a theorem as follows.

Theorem 2: For two shift-invariant subarrays with data correlation matrices given by $\mathbf{R}_{x i}$ and $\mathbf{R}_{x j}$, respectively, a noise eigenvector of $\mathbf{R}_{x i}$ is also a noise eigenvector of $\mathbf{R}_{x j}$.

Proof: Assume that the data vectors received by these two subarrays are given by

$$
X_{i}(t)=\mathbf{A}_{i}(\theta) S(t)+N_{i}(t)
$$

and

$$
X_{j}(t)=\mathbf{A}_{j}(\theta) S(t)+N_{j}(t)
$$

respectively. Using the shift-invariant property, (20) can be rewritten as

$$
X_{j}(t)=\mathbf{A}_{i}(\theta) \Phi S(t)+N_{j}(t)
$$

where $\Phi$ denotes a $J \times J$ diagonal phase matrix due to the shift between these two subarrays. $N_{i}(t)$ and $N_{j}(t)$ are the noise vectors received by these two subarrays, respectively. Let $u$ be a noise eigenvector of $\mathbf{R}_{x i}=E\left\{X_{i}(t) X_{i}^{H}(t)\right\}$. Then $u$ is orthogonal to $\mathbf{A}_{i}(\theta)$. It follows from (21) that

$$
\mathbf{R}_{x j} u=\left(\mathbf{A}_{i}(\theta) \Phi \mathbf{S} \Phi^{H} \mathbf{A}_{i}^{H}(\theta)\right) u+\sigma_{n}^{2} u=\sigma_{n}^{2} u
$$

where $\mathbf{R}_{x j}=E\left\{X_{j}(t) X_{j}^{H}(t)\right\}$. Equation (22) reveals that the noise eigenvector $u$ is also a noise eigenvector of $\mathbf{R}_{x j}$. This completes the proof.

Based on the above shift-invariance property, a matrix $\Pi=\left[\begin{array}{llll}\Pi_{1} & \Pi_{2} & \cdots & \Pi_{K}\end{array}\right]$ is further constructed with each submatrix $\Pi_{k}, k=1,2, \cdots, K$ given by

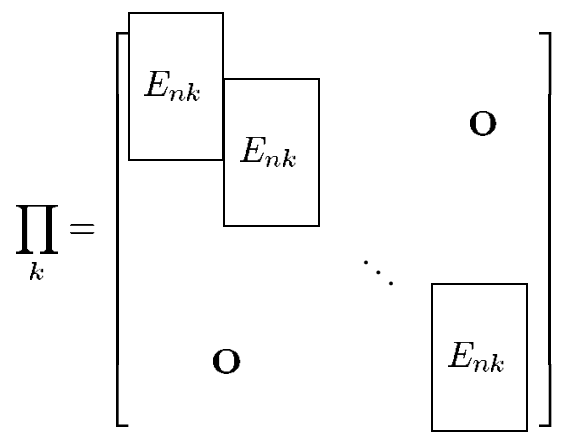

with size of $N \times\left(N_{k}-J\right)\left(N-N_{k}+1\right)$ for the ULA case, where $\mathbf{O}$ denotes a zero matrix with appropriate size. In (23), the $\mathbf{E}_{n k}$ in the $i$ th block starts from $\Pi_{k}\left(i, i\left(N_{k}-J\right)\right)$ to $\Pi_{k}\left(i+N_{k}, i N_{k}\right)$. Following the similar manner, one can form a similar matrix $\Pi=\left[\begin{array}{llll}\Pi_{1} & \Pi_{2} & \cdots & \Pi_{K}\end{array}\right]$ for an arbitrary array with multiple shift invariances. However, expressing $\Pi_{k}$ for $k=1,2, \cdots, K$ is not an easy task. Here, we only take a $6 \times 6$ URA for illustration. Consider that $J=8$ and each 2 -D subarray has size $3 \times 3$ as shown in Fig. 1(b). The noise subspace associated with each subarray contains only one noise eigenvector. Let the noise eigenvector be designated as $e_{n k}=\left[\begin{array}{llll}e_{n k}(1) & e_{n k}(2) & \cdots & e_{n k}(9)\end{array}\right]^{T}$ for the $k$ th subarray. Then $\Pi_{k}$ is given by (24), shown at the bottom of the page. The main difference between (23) and (24) is that we use $\mathbf{E}_{n k}$ to form the submatrix $\Pi_{k}$ for the ULA case, while using $\tau_{n k}$ to form $\Pi_{k}$ for the URA case.

It is easy to show that each column of $\prod$ is orthogonal to the signal subspace spanned by $\mathbf{E}_{s}$. Let the coefficient matrix $\mathbf{G}$ be expressed as $\mathbf{G}=\left[\begin{array}{llll}\mathbf{G}_{1}^{T} & \mathbf{G}_{2}^{T} & \cdots & \mathbf{G}_{K}^{T}\end{array}\right]^{T}$, where each of $\mathbf{G}_{k}, k=1,2, \cdots, K$ is a $J \times J$ matrix. Since $\mathbf{E}_{s k} \mathbf{G}_{k}$ and $\mathbf{A}_{k}(\theta)$ span the same signal subspace for the kth subarray, we can write $\mathbf{E}_{s k} \mathbf{G}_{k}=\mathbf{A}_{k}(\theta) \mathbf{T}_{k}$, where $\mathbf{T}_{k}$ is a $J \times J$ transformation matrix with rank $=J$. Hence, $\mathbf{G}_{k}=\mathbf{E}_{s k}^{H} \mathbf{A}_{k}(\theta) \mathbf{T}_{k}$. Next, $\mathbf{G}_{1}$ can be set to the identity matrix $\mathbf{I}$ without loss of generality. Then $\mathbf{G}=\left[\begin{array}{ll}\mathbf{I} & \overline{\mathbf{G}}^{T}\end{array}\right]^{T}$. Since $\Pi$ is orthogonal to the signal subspace spanned by $\mathbf{E}_{s}$, we have

$$
\Pi^{H} \mathbf{E}_{s}=\Pi^{H} \mathbf{Q}_{2} \mathbf{G}=\Psi \mathbf{G}=\left[\begin{array}{ll}
\Psi_{1} & \Psi_{2}
\end{array}\right]\left[\frac{\mathbf{I}}{\mathbf{G}}\right]=0
$$

where $\Psi_{1}$ contains the first $J$ columns of $\Psi=\Pi^{H} \mathbf{Q}_{2}$ whose size is $\sum_{k=1}^{K}\left(N_{k}-J\right)\left(N-N_{k}+1\right) \times(K J)$, while the other $(K-1) J$ columns of $\Psi$ are contained in $\Psi_{2}$. It follows from (25) that

$$
\Psi_{2} \overline{\mathbf{G}}=-\Psi_{1}
$$

Equation (26) reveals that the elements of $\overline{\mathbf{G}}$ can be obtained by solving a set of linear equations. The condition of $\sum_{k=1}^{K}\left(N_{k}-J\right)\left(N-N_{k}+1\right)>(K-1) J$ must be held in order to obtain a unique solution for $\overline{\mathbf{G}}$. Thus, the solution of $\overline{\mathbf{G}}$ can be found by using an LS method and is given by

$$
\overline{\mathbf{G}}=-\left(\Psi_{2}^{H} \Psi_{2}\right)^{-1} \Psi_{2}^{H} \Psi_{1}
$$

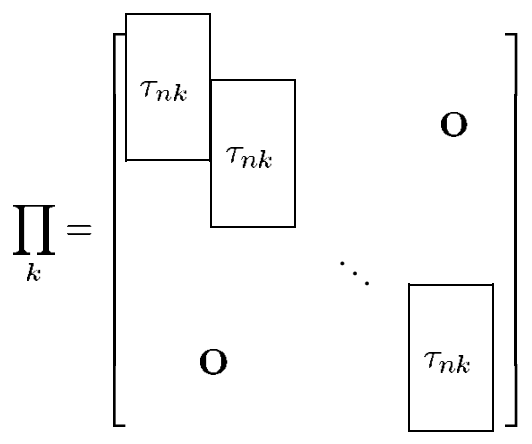

where

$$
\tau_{n k}=\left[e_{n k}(1) e_{n k}(2) e_{n k}(3) \quad 000 \quad e_{n k}(4) e_{n k}(5) e_{n k}(6) \quad 000 \quad e_{n k}(7) e_{n k}(8) e_{n k}(9)\right]^{T}
$$


However, the obtained eigensubspaces associated with each subarray may be perturbed due to the effect of finite data samples in practical applications. As a result, the matrix $\Psi$ may suffer from errors. To alleviate this difficulty, we resort to the TLS method for solving $\overline{\mathbf{G}}$. From the singular value decomposition (SVD) of the matrix $\left[\begin{array}{ll}\Psi_{2} & -\Psi_{1}\end{array}\right]$, we have [13]

$$
\left[\Psi_{2}-\Psi_{1}\right]=\mathbf{U}_{\Psi} \Sigma_{\Psi} \mathbf{V}_{\Psi}^{H}=\mathbf{U}_{\Psi} \Sigma_{\Psi}\left[\begin{array}{ll}
\mathbf{V}_{11} & \mathbf{V}_{12} \\
\mathbf{V}_{21} & \mathbf{V}_{22}
\end{array}\right]^{H}
$$

where $\mathbf{V}_{12}$ and $\mathbf{V}_{22}$ are two $((K-1) J) \times J$ and $J \times J$ matrices, respectively. From (26) and (28), the TLS solution for $\overline{\mathbf{G}}$ is given by [13]

$$
\overline{\mathbf{G}}=-\mathbf{V}_{12} \mathbf{V}_{22}^{-1} \text {. }
$$

Substituting (29) into $\mathbf{G}=\left[\begin{array}{ll}\mathbf{I} & \overline{\mathbf{G}}^{T}\end{array}\right]^{T}$ and performing the SVD on $\mathbf{G}$ yields

$$
\mathbf{G}=\left[\begin{array}{ll}
\mathbf{U}_{g s} & \mathbf{U}_{g n}
\end{array}\right] \Sigma_{g} \mathbf{V}_{g}^{H}=\left[\begin{array}{ll}
\mathbf{U}_{g s} & \mathbf{U}_{g n}
\end{array}\right]\left[\begin{array}{cc}
\Sigma_{g s} & \mathbf{O} \\
\mathbf{O} & \mathbf{O}
\end{array}\right] \mathbf{V}_{g}^{H}
$$

where $\mathbf{U}_{g s}$ contains the $J$ left-singular vectors with nonzero singular values in $\Sigma_{g s}$, while $\mathbf{U}_{g n}$ contains the other $(K-$ 1) $J$ left-singular vectors with zero singular values in $\Sigma_{g}$. Substituting (30) into (17) yields

$$
\tilde{\mathbf{E}}_{s}=\mathbf{Q}_{2} \mathbf{U}_{g s} .
$$

Moreover, based on the result of (30), we can show that (18) is given by

$$
\tilde{\mathbf{E}}_{n}=\left[\begin{array}{ll}
\mathbf{Q}_{1} & \mathbf{Q}_{2} \mathbf{U}_{g n}
\end{array}\right] .
$$

In the following, we present a procedure for implementing the proposed technique.

\section{Procedure:}

Step 1: Perform the EVD on the data correlation matrix $\mathbf{R}_{x k}$ of the $k$ th subarray obtained from the partition to get $\mathbf{E}_{s k}$ and $\mathbf{E}_{n k}$ for $k=1,2, \cdots, K$.

Step 2: Construct the matrices $\mathbf{Q}_{1}, \mathbf{Q}_{2}$, and $\prod$ using (11), (12), and (23), respectively.

Step 3: Construct the matrix $\Psi=\left[\begin{array}{ll}\Psi_{1} & \Psi_{2}\end{array}\right]=\Pi^{H} Q_{2}$.

Step 4: Obtain the matrix $\overline{\mathbf{G}}=-\left(\Psi_{2}^{H} \Psi_{2}\right)^{-1} \Psi_{2}^{H} \Psi_{1}$ if the LS solution is required or perform the SVD on the matrix $\left[\Psi_{2}-\Psi_{1}\right]$ to get $\left[\Psi_{2}-\Psi_{1}\right]=\mathbf{U}_{\Psi} \Sigma_{\Psi} \mathbf{V}_{\Psi}^{H}$ and then compute the matrix $\overline{\mathbf{G}}=-\mathbf{V}_{12} \mathbf{V}_{22}^{-1}$ if the TLS solution is required.

Step 5: Perform the SVD on the matrix $\mathbf{G}$ to get $\mathbf{G}=$ $\left[\begin{array}{ll}\mathbf{U}_{g s} & \mathbf{U}_{g m}\end{array}\right] \Sigma_{g} \mathbf{V}_{g}^{H}$ as shown in (30).

Step 6: Finally, form the matrices $\tilde{\mathbf{E}}_{s}=\mathbf{Q}_{2} \mathbf{U}_{g s}$ and $\tilde{\mathbf{E}}_{n}=$ $\left[\begin{array}{ll}\mathbf{Q}_{1} & \mathbf{Q}_{2} \mathbf{U}_{g n}\end{array}\right]$

Note that we only have to find the eigensubspaces for the first subarray when the number of array elements for each subarray is equal to $N / K$ (i.e., uniform partition). Then set $\mathbf{E}_{s k}=\mathbf{E}_{s 1}$ and $\mathbf{E}_{n k}=\mathbf{E}_{n 1}$ for $k=2,3, \cdots, K$ in the Step 1 of the above procedure to save computations.

Next, we consider the use of $\tilde{\mathbf{E}}_{s}$ and $\tilde{\mathbf{E}}_{n}$ obtained by using the proposed technique for the applications discussed in Section II.

\section{A. Eigenspace-Based Interference Cancellation}

For the application of ESB interference cancellation, we apply the above procedure to find the basis matrix $\tilde{\mathbf{E}}_{I}$ instead of $\tilde{\mathbf{E}}_{s}$, which spans the same interference subspace as that spanned by $\mathbf{E}_{I}$ in the incoherent signal situation. The optimal weight vector $W_{o}$ can be obtained from (6) by substituting $\tilde{\mathbf{E}}_{I}$ into (7). To cure the problem of coherent signals, we present a cross-correlation method based on the concept of [14] as follows. The desired signal contained in $X(t)$ is first removed by passing $X(t)$ through the signalblocking matrix $\mathbf{B}$ as given by (8) to obtain a data vector $Y(t)=\left[\begin{array}{llll}y_{1}(t) & y_{2}(t) & \cdots & y_{N-1}(t)\end{array}\right]^{T}=\mathbf{B}^{H} X(t)$. Then the following matrix is constructed

$$
\mathbf{P}_{y k}=E\left\{\left[\begin{array}{cccc}
y_{1}(t) & y_{2}(t) & \cdots & y_{J-1}(t) \\
y_{2}(t) & y_{3}(t) & \cdots & y_{J}(t) \\
\vdots & \vdots & \vdots & \vdots \\
y_{N_{k}}(t) & y_{N_{k}+1}(t) & \cdots & y_{N_{k}+J-2(t)}
\end{array}\right] y_{j}^{*}(t)\right\}
$$

where $y_{j}(t)$ is chosen such that its noise component is uncorrelated to the noise component of $y_{i}(t)$ for $i=1,2, \cdots, N_{k}+$ $J-2$, i.e., $j$ must be greater than $N_{k}+J-1$. Performing SVD on (33) yields

$$
\mathbf{P}_{y k}=\left[\begin{array}{ll}
\mathbf{E}_{s k} & \mathbf{E}_{n k}
\end{array}\right] \Sigma_{y k} \mathbf{V}_{y k}^{H}
$$

where $\mathbf{E}_{s k}$ denotes the $N_{k} \times(J-1)$ matrix spanning the interference subspace and $\mathbf{E}_{n k}$ the $N_{k} \times\left(N_{k}-J+1\right)$ matrix spanning the noise subspace associated with the $k$ th subarray. After obtaining $\mathbf{E}_{s k}$ and $\mathbf{E}_{n k}$, we utilize the proposed technique to construct the basis matrix $\tilde{\mathbf{E}}_{I}$, which spans the same interference subspace as that spanned by $X(t)$. Finally, we substitute $\tilde{\mathbf{E}}_{I}$ instead of $\mathbf{E}_{I}$ into (7) to find the optimal weight vector $\mathbf{W}_{o}$ as given by (6).

\section{B. Bearing Estimation}

In this case, we substitute $\tilde{\mathbf{E}}_{n}$ obtained by using the above procedure into (9) to form the spatial spectrum required by the MUSIC algorithm as follows:

$$
E(\theta)=\frac{1}{S^{H}(\theta) \tilde{\mathbf{E}}_{n} \tilde{\mathbf{E}}_{n}^{H} S(\theta)} .
$$

When coherent signals are present, we first utilize the spatial smoothing scheme to obtain the smoothed data correlation matrix for each of the $k$ th subarray, $k=1,2, \cdots, K$. Then the smoothed data correlation matrix is used to replace $\mathbf{R}_{x k}$. in Step 1 of the above procedure for finding the required $\mathbf{E}_{s k}$ and $\mathbf{E}_{n k}$.

\section{Evaluation of the ReQuired COMPUTATIONAL COMPLEXITY}

Here, we only consider the evaluation for a ULA with uniform partition, i.e., $N_{k}=N / K, k=1,2, \cdots, K$, in the incoherent signal environment for simplicity. Using shiftinvariant property leads to the fact that $\mathbf{E}_{s i}=\mathbf{E}_{s j}$ and $\mathbf{E}_{n i}=\mathbf{E}_{n j}$ for any two subarrays $i, j=1,2, \cdots, K$. We 
only have to compute the eigensubspaces of $\mathbf{R}_{x k}$ of the $k$ th subarray for any selected $k=1,2, \cdots, K$.

Based on the procedure presented in Section III, in the following we summarize the required complex multiplications $(C M)$ for finding the eigensubspaces spanned by $X(t)$.

In Step 1: Performing the EVD using the method presented in [13] on $\mathbf{R}_{x k}$ requires about $6(N / K)^{3} C M$ in order to find $\mathbf{E}_{s k}$ and $\mathbf{E}_{n k}$.

In Step 3: Since $\mathbf{E}_{n i}=\mathbf{E}_{n k}$ for any $i=1,2, \cdots, K$, when using multiple shift-invariant subarrays, we note from (23) that $\Pi_{i}=\Pi_{k}$ and, thus, $\Pi$ can be set to $\Pi_{1}$ for further saving $C M$. Hence, only the values of $2 J(N / K-J)$ different entries in $\Psi$ must be computed. This costs about $J(N / K)(N / K+1)(N / K-J) C M$.

In Step 4: If the LS solution is used, then computing the product $\Psi_{2}^{H} \Psi_{2}$ requires about $(K-1)^{2} J^{2}(N / K-J)(N-$ $N / K+1) C M$, while computing the product $\Psi_{2}^{H} \Psi_{1}$ costs about $(K-1) J^{2}(N / K-J)(N-N / K+1) C M$. Obtaining $\overline{\mathbf{G}}$ from (27) requires $(K-1)^{3} \boldsymbol{J}^{3}+(K-1)^{2} J^{3} C M$.

In Step 4: If the TLS solution is used, then performing the SVD of $\left[\begin{array}{ll}\Psi_{2} & -\Psi_{1}\end{array}\right]$ requires about $4(K J)^{2}(N / K-J)(N-$ $N / K+1)+8(K J)^{3} C M$, while computing the product $-\mathbf{V}_{12} \mathbf{V}_{22}^{-1}$ to obtain $\overline{\mathbf{G}}$ requires about $K(K-1) J^{3} C M$.

In Step 5: Performing the SVD of $\mathbf{G}$ requires about $4 K(K-2) J^{3} C M$.

In Step 6: Finally, computing the products $\mathbf{Q}_{2} \mathbf{U}_{g s}$ and $\mathbf{Q}_{2} \mathbf{U}_{g n}$ to obtain $\tilde{\mathbf{E}}_{s}$ and $\tilde{\mathbf{E}}_{n}$ costs $N J^{2} C M$.

Therefore, the total number of complex multiplications required for obtaining the basis matrices $\tilde{\mathbf{E}}_{s}$ and $\tilde{\mathbf{E}}_{n}$ is approximately given by

$$
C M_{l s}=(6+J)\left(\frac{N}{K}\right)^{3}+K J^{2} N^{2}+N J^{2}+(K J)^{3}
$$

if the LS solution for $\overline{\mathbf{G}}$ is employed. In contrast, based on conventional ESB methods which directly perform the EVD of $\mathbf{R}_{x}$, the number of the $C M$ required is about [13] $C M_{f}=6 N^{3}$ in order to obtain the basis matrices $\mathbf{E}_{s}$ and $\mathbf{E}_{n}$. Taking the ratio of $C M_{l s}$ and $C M_{f}$ yields

$$
\begin{aligned}
\frac{C M_{l s}}{C M_{f}} & =\left(1+\frac{J}{6}\right)\left(\frac{1}{K}\right)^{3}+\frac{K J^{2}}{6 N}+\frac{1}{6}\left(\frac{K J}{N}\right)^{3}+\frac{1}{6}\left(\frac{J}{N}\right) \\
& \approx\left(1+\frac{J}{6}\right)\left(\frac{1}{K}\right)^{3}
\end{aligned}
$$

if $6 N \gg K J^{2}$. Next, the total number of complex multiplications required for obtaining the basis matrices $\tilde{\mathbf{E}}_{s}$ and $\tilde{\mathbf{E}}_{n}$ is approximately given by

$$
C M_{t l s}=(6+J)\left(\frac{N}{K}\right)^{3}+5 K J^{2} N^{2}+N J^{2}+9(K J)^{3}
$$

if the TLS solution for $\overline{\mathbf{G}}$ is employed. Again, taking the ratio of $C M_{t l s}$ and $C M_{f}$ yields

$$
\begin{aligned}
\frac{C M_{t l s}}{C M_{f}} & =\left(1+\frac{J}{6}\right)\left(\frac{1}{K}\right)^{3}+\frac{5 K J^{2}}{6 N}+\frac{3}{2}\left(\frac{K J}{N}\right)^{3}+\frac{1}{6}\left(\frac{J}{N}\right)^{2} \\
& \approx\left(1+\frac{J}{6}\right)\left(\frac{1}{K}\right)^{3}
\end{aligned}
$$

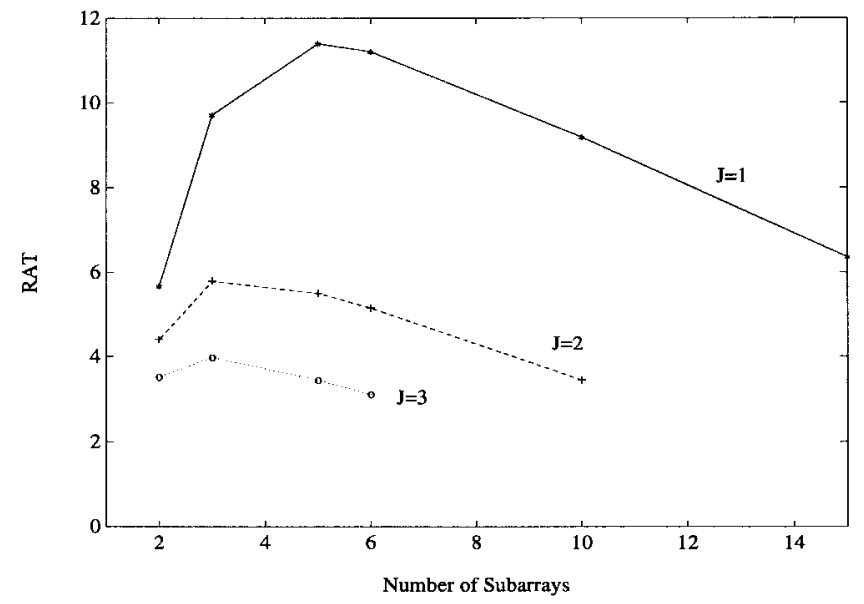

Fig. 2. The ratio of the amounts of flops using the conventional ESB and the proposed techniques.

if $6 N \gg K J^{2}$. From (37) and (39), we note that the computational complexity required by the proposed technique is approximately equal to $1 / K^{3}$ of that required by existing ESB methods when the number $N$ of the array elements is large enough. To further show the computational saving for an array with $N$ not large enough, we here perform the simulations on the software implementation of the conventional ESB and the proposed techniques using the MATLAB programming system. Fig. 2 plots the curves showing the comparison for the case of using a ULA with $N=30$ and $J=1,2$, and 3 . The RAT is defined as the ratio of the amounts of the flops required by these two techniques. We note that the proposed technique significantly reduces the required flops, especially the ratio is over 11 when the number of subarrays used is five in the case of $J=1$.

Considering the URA case, we can follow the similar manner as for the ULA case to compare the complex multiplications required by the proposed and existing ESB techniques. Our experience shows that the proposed technique also costs less computational complexity than the existing ESB techniques. However, the evaluation is very complicated for the URA case and is omitted.

\section{COMPUTER Simulation EXAMPLES}

Several simulation examples are presented in this section for illustrating the effectiveness of the proposed technique. We consider both the cases of using ULA and URA. For all simulations, the array interelement spacing is set to one half of the signal wavelength. The ensemble correlation matrix $\mathbf{R}_{x}$ is replaced by the sample correlation matrix $\hat{\mathbf{R}}_{x}$ given by $\hat{\mathbf{R}}_{x}=\sum_{t=1}^{L} X(t) X^{H}(t) / L$, where $L$ is the number of data snapshots. The original whole array is uniformly partitioned into $K$ subarrays, i.e., $N_{k}=N / K$ for $k=1,2, \cdots, K$.

1) Eigenspace-Based Interference Cancellation: First, in the case of coherent signals, an interference canceller using a ULA with $N=24$ is considered. The desired signal with $\mathrm{SNR}=0 \mathrm{~dB}$ is impinging on the array from the broadside, while three coherent interferers with equal INR $=30 \mathrm{~dB}$ are impinging on the array from 20,30 , and -15 degrees, respectively. Fig. 3 shows the array output SINR versus 


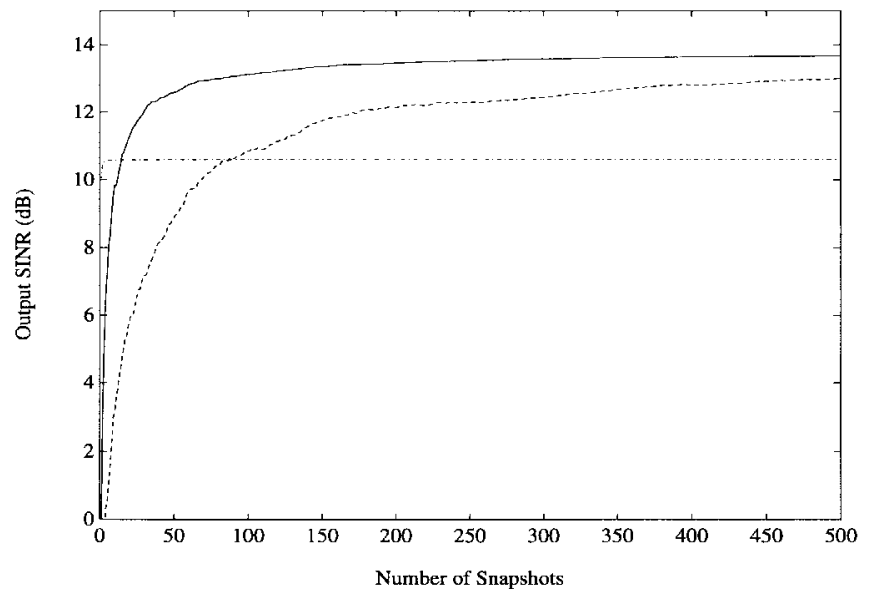

Fig. 3. The output SINR versus the number of snapshots. The proposed technique using two subarrays (solid curve) and three subarrays (dash curve). Dash-dot curve: the eigenspace-based interference canceller with spatial smoothing.

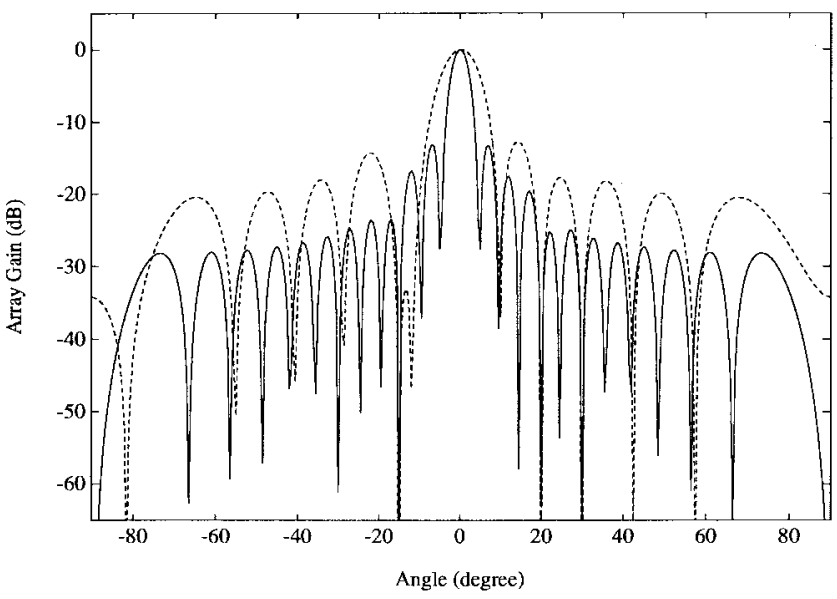

Fig. 4. The output beampatterns. Solid curve: the proposed technique using two subarrays. Dash curve: the eigenspace-based interference canceller with spatial smoothing.

the number of snapshots using the proposed technique in conjunction with the proposed cross-correlation method as described in (33) and (34) with $N_{k}=8$ and 12, while Fig. 4 shows the corresponding array output beampatterns with $L=500$. The simulation results of using the eigenspace-based interference canceller of [6] are also given for comparison. To eliminate the coherent interferers, we incorporate the interference canceller with the spatial smoothing scheme based on 12-element subarrays. These two figures show that the eigenspace-based interference canceller of [6] possesses faster convergence speed. However, the proposed technique provides better steady-state performance because it does not sacrifice the degrees of freedom available from the original array. The simulation result of the output SINR versus the number $K$ of subarrays is plotted in Fig. 5. Again, as expected, the array performance degrades as $K$ increases since the degrees of freedom available for extracting the noise subspace of the subarray reduces as $K$ increases.

Next, we consider the use of eigenspace-based interference cancellers in the incoherent signal situation. The array scenario and signal characteristics are the same as those for the above

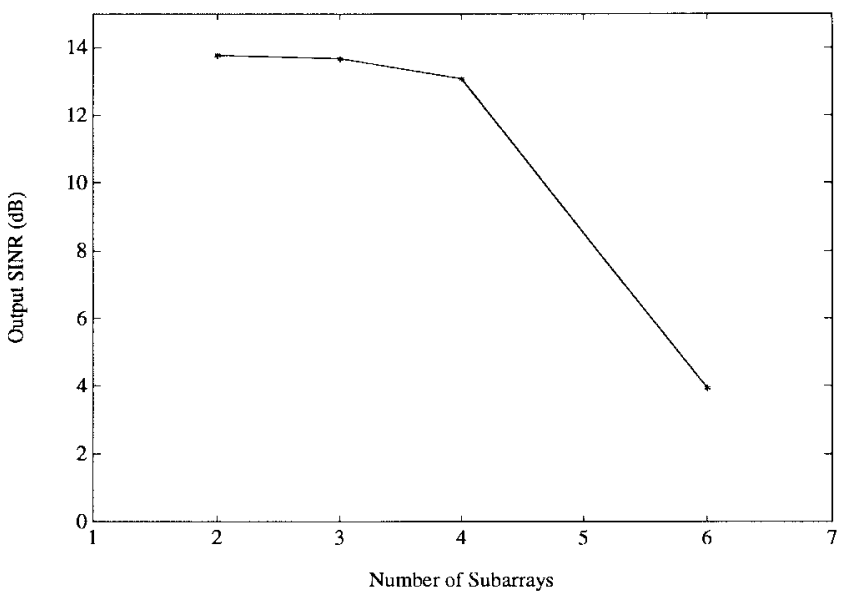

Fig. 5. The output SINR versus the number of subarrays.

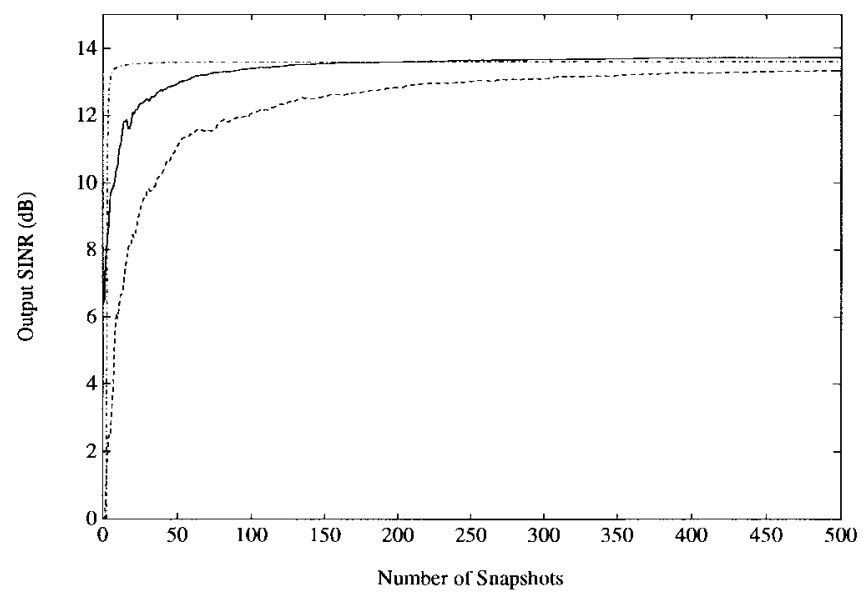

Fig. 6. The output SINR versus the number of snapshots. The proposed technique using two subarrays (solid curve) and three subarrays (dash curve). Dash-dot curve: the eigenspace-based interference canceller.

coherent case except that the three interferers are now incoherent. Fig. 6 shows the array output SINR versus the number of snapshots using the eigenspace-based interference canceller of [6] and the proposed technique with $N_{k}=8$ and 12 . We note that using the proposed technique provides satisfactory convergence speed and array performance. Each of the above simulation results is obtained by taking the average of 100 independent runs.

2) Bearing Estimation: First, the bearing estimation of coherent signals using a ULA with $N=24$ is considered. Three coherent signals with equal SNR $=10 \mathrm{~dB}$ are impinging on the array from $-5^{\circ},-2^{\circ}$, and $5^{\circ}$, respectively. Fig. 7 shows the spatial spectra of using the proposed technique with $N_{k}=8$, subarray size $=8$ for employing the spatial smoothing scheme, and the TLS solution for finding the matrix $\overline{\mathbf{G}}$. The spatial spectra of using the conventional MUSIC algorithm with and without the spatial smoothing scheme based on eight-element subarrays are also given for comparison. The number of data snapshots used is 100 . From this figure, we observe that the proposed technique provides better resolution capability. This is the expected result from the fact that unlike the conventional MUSIC algorithm, the proposed technique does not sacrifice the degrees of freedom in extracting the noise subspace when 


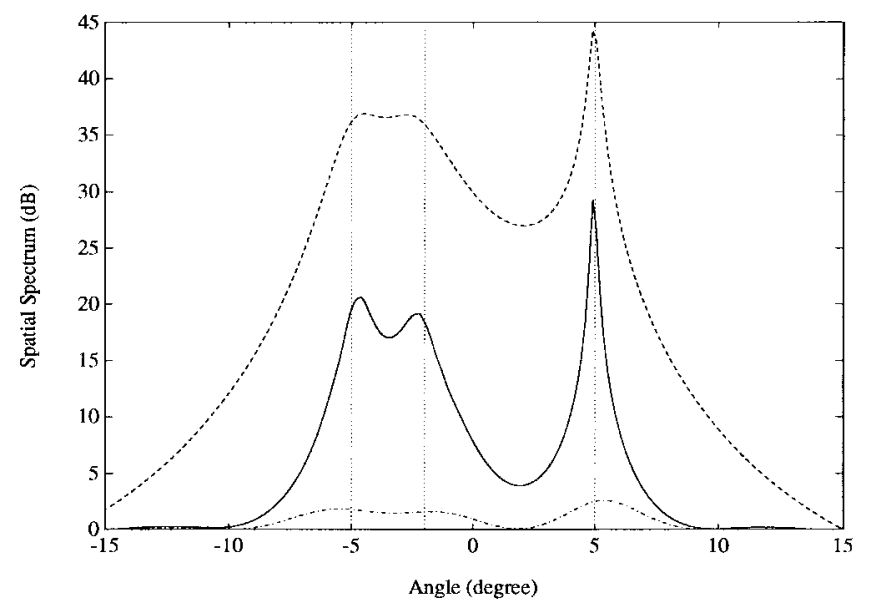

Fig. 7. The spatial spectra. Solid curve: the proposed technique using three subarrays. Dash curve: the MUSIC algorithm with spatial smoothing. Dash-dot curve: the MUSIC algorithm without spatial smoothing.

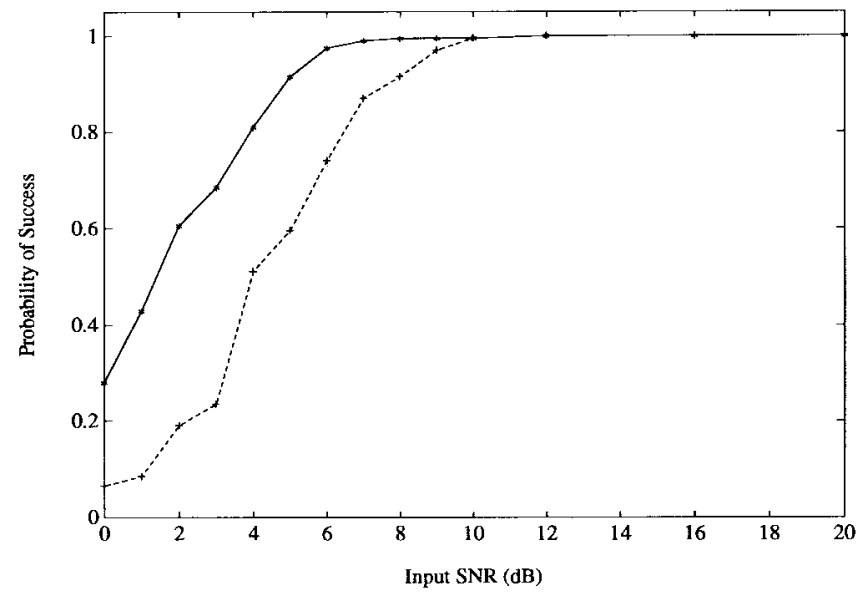

Fig. 8. The probability of successfully resolving two signals versus the input SINR. Solid curve: the proposed technique using two subarrays. Dash curve: the MUSIC algorithm.

using the spatial smoothing scheme to tackle the problem of signal coherence. Each of the above simulation results is obtained by taking the average of 100 independent runs.

Next, we use a ULA with $N=20$ to estimate the bearings of two incoherent signals with direction angles $=$ $-2^{\circ}$ and $2^{\circ}$, respectively. Fig. 8 shows the probabilities for successfully resolving the signals under different input SNR using the proposed technique with two ten-element subarrays and the conventional MUSIC algorithm, while Table I lists the estimation bias and mean-square error. For this case, 200 independent runs are performed and the probability is defined as the number of successes in resolving two signals divided by 200 . We note that the proposed technique can resolve the signals with higher probability and less bias.

Finally, we consider the 2-D bearing estimation of coherent signals using a $6 \times 6$ URA. Three coherent signals with equal $\mathrm{SNR}=20 \mathrm{~dB}$ are impinging on the 2-D array from $(u, v)=$ $(0.4,0.1),(0.25,0.35)$, and $(0.15,0.15)$, respectively, where $u=\sin (\theta) \cos (\phi)$ and $v=\sin (\theta) \sin (\phi)$. For each signal source, its elevation and azimuth angles are designated as $\theta$ and $\phi$, respectively. We partition the $6 \times 6$ URA into uniform rectangular $3 \times 3$ subarrays $\left(N_{k}=9\right)$ when using
TABLE I

The Estimation Bias and Mean Square ERROR (MSE) FOR BEARING ESTIMATION

\begin{tabular}{c|c|c|c|c||c|c|c|c}
\cline { 2 - 9 } & \multicolumn{3}{c||}{ The Proposed Techrique } & \multicolumn{3}{c}{ The Conventional MUSIC Algorithm } \\
\cline { 2 - 9 } & \multicolumn{2}{c}{ Bias } & \multicolumn{2}{c|}{ MSE } & \multicolumn{2}{c|}{ Bias } & \multicolumn{2}{c}{ MSE } \\
\hline SNR $(\mathrm{dB})$ & $-2^{\circ}$ & $2^{\circ}$ & $-2^{\circ}$ & $2^{\circ}$ & $-2^{\circ}$ & $2^{\circ}$ & $-2^{\circ}$ & $2^{\circ}$ \\
\hline 2 & 0.0554 & 0.0012 & 1.1530 & 1.5289 & 0.3676 & -0.3795 & 0.4117 & 0.4657 \\
6 & 0.1078 & -0.0519 & 0.4793 & 0.4550 & 0.3511 & -0.3052 & 0.2677 & 0.2239 \\
10 & -0.0143 & -0.0466 & 0.0964 & 0.1117 & 0.1240 & -0.1490 & 0.0498 & 0.0673 \\
16 & -0.0018 & -0.0160 & 0.0169 & 0.0165 & 0.0286 & -0.0471 & 0.0105 & 0.0118 \\
20 & 0.0025 & -0.0008 & 0.0062 & 0.0062 & 0.0132 & -0.0114 & 0.0036 & 0.0034 \\
\hline
\end{tabular}

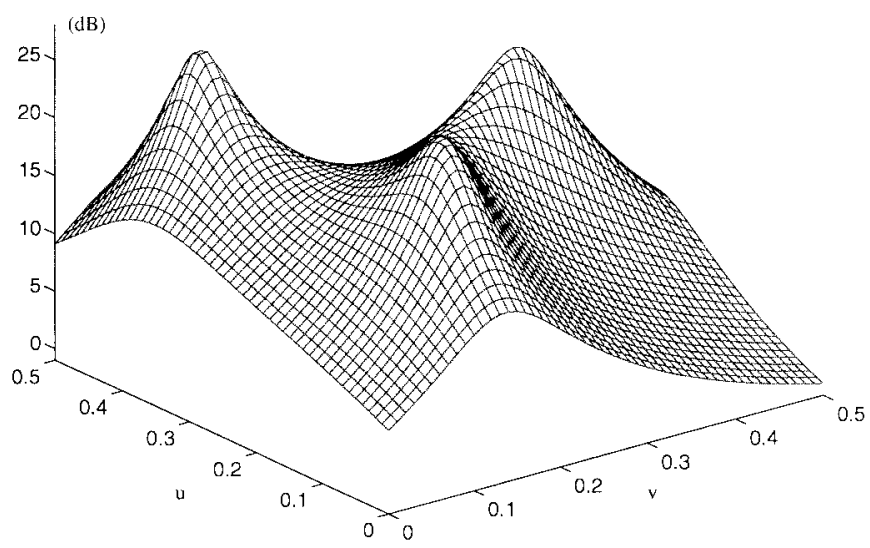

(a)

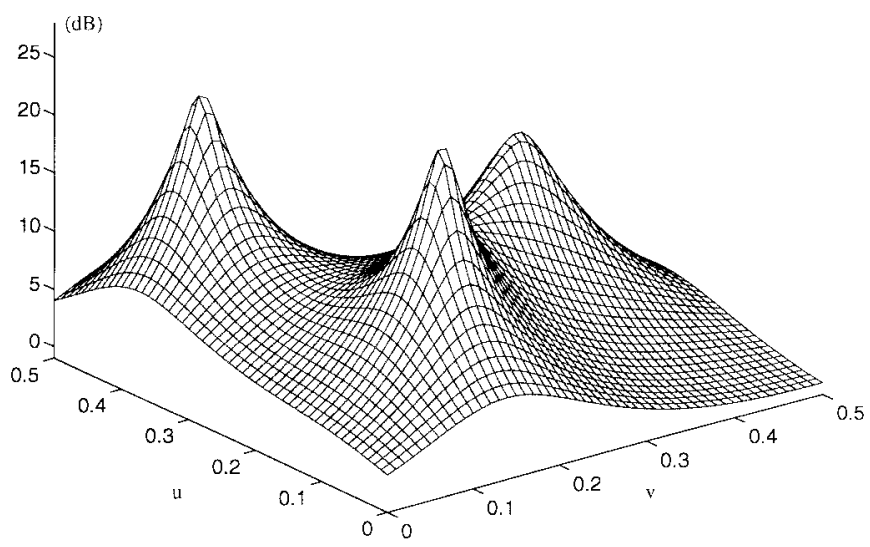

(b)

Fig. 9. (a) The 2-D spatial spectrum using the MUSIC algorithm with spatial smoothing. (b) The 2-D spatial spectrum using the proposed technique.

the proposed technique in conjunction with the 2-D spatial smoothing scheme of [11] based on $3 \times 3$ subarrays. Fig. 9 plots the simulation results after 300 data snapshots. For comparison, the simulation using the conventional MUSIC algorithm with the spatial smoothing scheme based on $3 \times$ 3 subarrays is also performed. Again, we note that the 2-D spatial spectrum of using the proposed technique shows better resolution than that of using the conventional MUSIC algorithm.

\section{CONCLUSION}

This paper has presented an efficient technique for eigenspace-based array signal processing. Utilizing the multiple shift-invariant property of an $N$-element array and 
partitioning it into $K$ subarrays with multiple shift invariances, the problem of finding the eigensubspaces spanned by the array data vector has been formulated as a least-squares or a total least-squares problem. The theoretical result shows that the required computational complexity is significantly reduced to $O\left((N / K)^{3}\right)$ when $6 N \gg K J^{2}$ for $J$ signal sources. Simulation examples have been provided for illustrating the computational savings. In conjunction with the spatial smoothing scheme for bearing estimation or the proposed cross-correlation method for interference cancellation, the proposed technique can effectively deal with the case of coherent signals without sacrificing the available degrees of freedom. Computer simulations show that the proposed technique demonstrates better performance as compared to the existing methods. Although the proposed technique possesses several advantages over the existing techniques, it is worth further investigating its sensitivity to perturbations in sensor locations which may occur in practical applications.

\section{REFERENCES}

[1] R. O. Schmidt, "Multiple emitter location and signal parameter estimation," IEEE Trans. Antennas Propagat., vol. AP-34, pp. 276-280, Mar. 1986.

[2] R. Roy and T. Kailath, "ESPRIT-Estimation of signal parameters via rotation invariance techniques," IEEE Trans. Acoust., Speech, Signal Processing, vol. 37, pp. 984-995, July 1989.

[3] A. L. Swindlehurst, B. Ottersten, R. Roy, and T. Kailath, "Multiple invariance ESPRIT," IEEE Trans. Acoust., Speech, Signal Processing, vol. 40, pp. 867-881, Apr. 1992.

[4] L. Chang and C.-C. Yeh, "Performance of DMI and eigenspace-based beamformers," IEEE Trans. Antennas Propagat., vol. 40, pp. 1336-1347, Nov. 1992.

[5] B. D. Van Been, "Eigenstructure based partially adaptive array design," IEEE Trans. Antennas Propagat., vol. 36, pp. 357-362, Mar. 1988.

[6] A. M. Haimovich and Y. Bar-Ness, "An eigenanalysis interference canceller," IEEE Trans. Signal Processing, vol. 39, pp. 76-84, Jan. 1991.

[7] B. Widrow and S. D. Stearns, Adaptive Signal Processing. Englewood Cliffs, NJ: Prentice-Hall, 1985.

[8] T.-J. Shan and T. Kailath, "Adaptive beamforming for coherent signals and interference," IEEE Trans. Acoust., Speech, Signal Processing, vol. ASSP-33, pp. 527-536, June 1985.

[9] T.-J. Shan, M. Wax, and T. Kailath, "On spatial smoothing for estimation of coherent signals," IEEE Trans. Acoust., Speech, Signal Processing, vol. ASSP-33, pp. 806-811, Aug. 1985.
[10] S. U. Pillai and B. H. Kwon, "Forward/backward spatial smoothing techniques for coherent signal identification," IEEE Trans. Acoust. Speech, Signal Processing, vol. 37, pp. 8-15, Jan. 1989.

[11] C.-C. Yeh, J.-H. Lee, and Y.-M. Chen, "Estimating two-dimensional angles of arrival in coherent source environment," IEEE Trans. Acoust. Speech, Signal Processing, vol. 37, pp. 153-155, Jan. 1989.

[12] Y. Bresler, V. U. Reddy, and T. Kailath, "Optimum beamforming for coherent signals and interference," IEEE Trans. Acoust., Speech, Signal Processing, vol. 36, pp. 833-843, June 1988.

[13] G. H. Golub and C. F. Van Loan, Matrix Computations. Baltimore, MD: Johns Hopkins Univ. Press, 1983.

[14] A. K. Luthra, "A solution to the adaptive nulling problem with a lookdirection constraint in the presence of coherent jammers," IEEE Trans. Antennas Propagat., vol. AP-34, pp. 702-710, May 1986.

Shiann-Jeng Yu was born in Tainan, Taiwan, on May 16, 1967. He received the B.S. degree from Chung Yuan Christian University, Chung Li, Taiwan, in 1989, and the M.S. degree from the National Taiwan University, Taipei, in 1991, all in electrical engineering.

He currently works for the National Space Program Office (NSPO) in Taiwan. His interests include adaptive signal processing, array signal processing, and satellite communication.

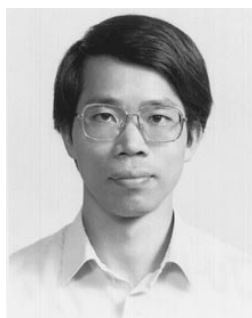

Ju-Hong Lee was born in I-Lan, Taiwan, on December 7, 1952. He received the B.S. degree from the National Cheng-Kung University, Tainan, Taiwan, in 1975, the M.S. degree from the National Taiwan University, Taipei, Taiwan, in 1977, and the Ph.D. degree from Rensselaer Polytechnic Institute, Troy, NY, in 1984, all in electrical engineering.

From September 1980 to July 1984, he was a Research Assistant and was involved in research on multidimensional recursive digital filtering in the Department of Electrical, Computer, and Systems Engineering at Resselaer Polytechnic Institute. From August 1984 to July 1986 he was a Visiting Associate Professor and later in August 1986 became an Associate Professor in the Department of Electrical Engineering, National Taiwan University. Since August 1989 he has been a Professor at the same university. He was appointed Visiting Professor in the Department of Computer Science and Electrical Engineering, University of Maryland, Baltimore, during a sabbatical leave in 1996. His current research interests include multidimensional signal processing, image processing, detection, and estimation theory, analysis and processing of joint vibration signals for the diagnosis of cartilage pathology, and adaptive signal processing and its applications in communications.

Dr. Lee received Outstanding Research Awards from the National Science Council in the academic years of 1988, 1989, and 1991-1994. 